\title{
Aplicación de un método multicriterio en la enseñanza de la investigación de mercados internacionales
}

\author{
Diego A. López-Cadavid(1), Juan G. Vanegas-López ${ }^{(1) \star}$ y José J. Baena-Rojas ${ }^{(2)}$ \\ (1) Facultad de Ciencias Administrativas y Económicas, Tecnológico de Antioquia Institución Universitaria, \\ Cl. 78b \#72A-220, Medellín, Colombia. (e-mail: diegolopezcadavid@gmail.com; jvanegas1@tdea.edu.co) \\ (2) Fundación Universitaria CEIPA, Escuela de Administración, Calle 77 Sur No. 40 - 165, Sabaneta, Colombia. \\ (e-mail: jose.baena@ceipa.edu.co)
}

${ }^{*}$ Autor a quien debe ser dirigida la correspondencia

Recibido Jul. 5, 2019; Aceptado Ago. 27, 2019; Versión final Sep. 25, 2019, Publicado Feb. 2020

\begin{abstract}
Resumen
El estudio presenta la aplicación de una metodología que busca facilitar la enseñanza sobre la selección de mercados internacionales, enfocada en la internacionalización de empresas. Se analiza la literatura existente que valida este tipo de enfoque para la toma de decisiones, identificando los factores generales que influyen en la selección de nuevos mercados de exportación. Luego se propone un nuevo factor de tipo económico con una serie de sub-factores a analizar, tales como riesgo en país, índice de precios al consumidor, PIB per cápita y tasa de desempleo. Se desarrolla la técnica de proceso analítico jerárquico para establecer la importancia relativa de los juicios emitidos por diez empresas del sector químico para cada una de las dimensiones previamente establecidas. Los resultados sugieren que los factores costos, logística, barreras comerciales, económico, y entorno y cultura son tenidos en cuenta en este orden importancia para la exportación de productos químicos desde Colombia; mientras que los mercados más atractivos para este caso son Estados Unidos, Honduras y Ecuador.
\end{abstract}

\section{Application of a multi-criteria approach in the teaching of international market research}

\begin{abstract}
The aim of this paper is to present the application of a methodology that seeks facilitating the teaching about the selection of international markets, focused on firm internationalization. The existing literature that validates this type of approach for decision-making is analyzed, identifying the general factors that have influence on the selection of new export markets. Then a new economic factor is proposed with a series of sub-factors to be analyzed, such as country risk, consumer price index, GDP per capita and the unemployment rate. The analytic hierarchy process technique was developed in order to rank the judgments made by ten chemical companies for each of the previously established dimensions. The results suggest that factors such as costs, logistics, trade barriers, economics, and environment and culture are taken into account, in this order of importance, for the exportation of chemical products from Colombia; while the most attractive markets for this case are the United States, Honduras and Ecuador.
\end{abstract}




\section{INTRODUCCIÓN}

En la actualidad, la internacionalización es un proceso que se encuentra ligado al mundo empresarial, donde las organizaciones deben gestionar diversos tipos de actividades y adaptarse a las condiciones del entorno, con el objetivo no solo de alcanzar nuevos mercados a nivel global (Barber y Darder, 2004), sino también de mejorar el desarrollo financiero de las empresas que implementen este proceso (London, 2010). De esta manera, es importante destacar que dichas transformaciones se ven fundamentadas e impulsadas por la constante búsqueda de mercados que representen mayores beneficios para un intercambio comercial y tengan condiciones adecuadas para una competencia equilibrada (Lanzas y Moral, 2010). En consecuencia, las empresas que deciden hacer presencia en mercados en el exterior generalmente poseen mayores beneficios respecto a aquellas empresas que solo buscan abastecer la demanda interna (Kox et al., 2010). Luego, las empresas que buscan obtener rentabilidad a partir del comercio internacional necesitan desarrollar actividades para mejorar su competitividad, cumplir con los estándares exigidos en otros mercados y superar las posibles adversidades que se presenten en éstos (Targhi et al., 2017). En este sentido, el contexto internacional actual conlleva a que las empresas busquen generar valor agregado, a través del desarrollo de capacidades y aptitudes del recurso humano de la organización, que posteriormente se traducen en ventajas sobre la competencia, enfocándose en la consecución de nuevos mercados y el mejoramiento de los mercados ya existentes (Leandro, 2009).

De esta forma, la toma de decisiones en el contexto internacional es un factor determinante que aumenta o disminuye la probabilidad de éxito que las empresas tendrían al competir en los mercados foráneos, siendo inevitable la adopción de distintas estrategias que apunten al aumento de las probabilidades de elegir un mercado óptimo para su incursión (Vanegas et al., 2018). Asimismo, cabe resaltar que el éxito en los mercados externos depende de la previa investigación y análisis de los posibles mercados a los cuales se podría exportar (Miečinskienè et al., 2014), siendo necesario para ello el estudio de las condiciones favorables que cada mercado puede ofrecer para satisfacer las necesidades a nivel empresarial (Chen y Wang, 2010; Cameron et al., 2017). Dicho análisis, debe tener como objeto la identificación de las oportunidades y posibles riesgos que se puedan ocasionar en cada mercado, buscando desvelar las ventajas y desventajas que estos representarían para las empresas (Barber y Darder, 2004). Por lo tanto, es recomendable que el mercado que se seleccione tenga cualidades similares, generando mejores condiciones ante una posible incursión, con el fin de que las empresas a partir de esta información puedan definir factores como: producto de entrada, entorno, competencia, precio y características distintivas (Akgün et al., 2014). De este modo, ha de señalarse que las empresas en general que desean internacionalizarse requieren de factores puntuales organizacionales que dependen de las características gerenciales de quienes administran, de variables del entorno relacionadas con el tipo de negocio en marcha como también de los recursos mismos disponibles de la firma (Vanegas et al., 2018); recursos que pueden facilitar la implementación y adquisición de tecnologías de información y otras a fines que pueden ayudar a darle mayor visibilidad a la organización a nivel internacional, además de promover el mejoramiento constante de diversos procesos productivos potenciales e incluso potencializar el acceso e intercambio de información para la consecución de contratos con proveedores y compradores en otros mercados alrededor del mundo (Bayo-Moriones et al., 2013).

Luego, se puede afirmar que la tasa de supervivencia en mercados foráneos con la internacionalización de las empresas depende en gran medida de la apropiada evaluación y toma de decisiones dentro del proceso integral de selección de mercados internacionales (SMI) (Papadopoulos y Denis, 1988; Rahman, 2006; Chen y Wang, 2010). Por esta razón, el presente artículo se torna relevante, ya que emplea una técnica rigurosa y pertinente en su implementación, la cual puede ser adoptada dentro del proceso de formación en educación superior para la SMI, a partir de un contexto en donde el mercado busca profesionales idóneos con un perfil altamente cualificado en un entorno global (Ruíz, 2013). Así pues, el presente método multicriterio otorga confiabilidad en el proceso de toma de decisiones dentro de la internacionalización de las empresas y a su vez cualifica a los estudiantes y futuros empresarios en técnicas ingenieriles para la toma de decisiones (Villalobos-Abarca et al., 2016), permitiendo sucesivamente el mejoramiento de competencias puntuales y específicas alusivas a la planeación, recolección y análisis las cuales no solo enriquecen el aprendizaje de mejores prácticas a nivel académico sino que además permiten corregir ciertas falencias durante procesos puntuales lo que se termina reflejando en el desarrollo de habilidades en el perfil de los profesionales (Martelo et al., 2018).

En consecuencia, con lo previamente resaltado por los distintos autores, contar con una herramienta de apoyo en la selección del destino ideal, resulta preponderante para la toma de decisiones referentes a la internacionalización y la selección de mercados, aplicando una ponderación jerárquica de los factores y subfactores, con el fin de obtener un puntaje o indicador para analizar si éste es viable frente a los distintos tipos de mercados (Cano et al., 2017; Baena et al., 2018). Observando dicha herramienta desde el punto de vista pedagógico, resalta el hecho de que esta metodología fortalece los procesos de enseñanza en investigación de mercados y marketing internacional, teniendo en cuenta que los modelos tradicionales de enseñanza en ocasiones carecen de efectividad debido a su falta de aplicabilidad en la realidad contextual de las empresas, 
haciendo necesario el uso de una metodología experimental para mejorar el aprendizaje de los estudiantes y desarrollar nuevas habilidades prácticas (Li et al., 2013). Cabe anotar que lo anterior no se traduce en que los elementos teórico-conceptuales sean inoperantes, ya que según De La Hoz et al. (2016) cada vez más el estudio de metodologías para analizar y medir el potencial exportador de las organizaciones en general toma más relevancia dada la formalización de criterios comunes que se hacen determinantes a nivel estratégico en la internacionalización; aunque en cualquier caso tales elementos deben complementarse con estrategias pedagógicas que estén enfocadas en la adquisición de aptitudes como el razonamiento crítico, el uso de los recursos tecnológicos y la resolución de problemas de manera creativa (Senna et al., 2013), acompañadas de un proceso investigativo que contraste los resultados con lo expuesto por las principales teorías de los negocios internacionales y motive a los estudiantes a continuar resolviendo distintos problemas en este campo (Fuerst, 2010) e incentive a los profesores a desarrollar métodos de enseñanza más efectivos (Crittenden y Wilson, 2006). Cabe resaltar también, que estas habilidades van ligadas a las expectativas que tiene el alumnado por adquirir las competencias suficientes para sobrevivir a los constantes cambios en la sociedad y la economía (Muduli et al., 2018), considerando que muchos no tienen la capacidad de costear una pasantía internacional o un programa especial que los ayude a adquirirlas (Kaufman et al., 2011), teniendo como objetivo final su aplicación en entornos empresariales y la generación de impacto en el ambiente de los negocios a nivel internacional (Vielba y Edelshain, 1995).

En síntesis, este estudio busca desarrollar una metodología pedagógica como estrategia para la enseñanza de los negocios internacionales, cuyos fines son: identificar las variables y sub-variables intrínsecas en la toma de decisiones para la internacionalización, proponer una técnica bajo múltiples criterios para valorar dichas variables, aplicar la técnica en un escenario real de exportación, y para hacerla operativa, se toma como estudio de caso el sector químico del departamento de Antioquia (Colombia), y por último, determinar los mercados actuales que presentan mayores ventajas para el sector, según la experiencia que los empresarios han tenido en las dimensiones identificadas. Así pues, la estructura de este estudio se divide en 4 partes, dando inicio con esta introducción que contextualiza la importancia de la expansión internacional, las estrategias para la selección de mercados y su enseñanza como ejemplo de aplicación contextualizada en escenarios empresariales y de negociación internacional. La segunda parte, destaca la propuesta metodológica del estudio, el método de recolección de la información y la aplicación del proceso analítico jerárquico (AHP). En la tercera parte, se destacan los resultados y el análisis obtenido, a partir del estudio de caso del sector químico desde la perspectiva de los negocios internacionales. Finalmente, en la cuarta parte las conclusiones destacan los hallazgos a nivel general, la importancia de la enseñanza de la metodología, su aplicabilidad a otros sectores y sus futuras líneas de investigación.

\section{PROPUESTA METODOLÓGICA}

Considerando la importancia de la internacionalización empresarial, esta propuesta establece una forma de abordar la enseñanza práctica de la investigación de mercados internacionales, proponiendo la técnica AHP en el sector químico antioqueño, pero considerando que la metodología puede ser extrapolable a distintos sectores debido a su practicidad y a las ventajas que acarrea para los empresarios que decidan aplicarla antes de tomar una decisión en un contexto global. De este modo, con esta metodología se busca promover el desarrollo de habilidades investigativas en los estudiantes del área de Negocios Internacionales y afines, a través de la contextualización aplicada de una técnica de toma de decisiones, el cual se encuentra dividido en 5 etapas: estructuración de las dimensiones a evaluar, identificación de las prioridades empresariales, proceso de selección de la muestra, recolección de la información, y, por último, determinación de los mercados más viables.

\section{Estructuración de las dimensiones a evaluar}

Para la implementación de ésta metodología, fue necesario diseñar y estructurar un esquema robusto, fiable y objetivo que abarcara todos los aspectos y dimensiones que teóricamente intervienen en la internacionalización de las empresas; dado que los factores y sub-factores que potencialmente pueden ser tenidos en cuenta suelen estar relacionados con aspectos de índole cultural, geográficos, económicos, políticos y sociales en general que evidencian la posible distancia entre un país respecto a otro, lo cual subsecuentemente puede resultar decisivo para la SMI (Martín y Drogendijk, 2014).

De esta manera, inicialmente se tomaron las 4 dimensiones y 11 variables previamente propuestas por Baena et al. (2018); no obstante, con el objetivo de robustecer esta aproximación, se adiciona el factor económico junto con 4 variables específicas que lo conforman, considerando su incidencia al analizar posibles compradores (Wang y Le, 2018). Así estas variables o sub-factores subyacentes influyentes según la literatura, pueden ser: el riesgo en país (Brouthers y Hennart, 2007), el índice de precios al consumidor (Canova y De Nicolo, 2002), el PIB per cápita (Cano et al., 2018) y la tasa de desempleo (Nakos y Brouthers, 2005). Asimismo son considerados adicionalmente nuevos sub-factores, respecto a la propuesta inicial, como 
el transporte interno de origen (Cipoletta et al., 2010), el tipo de cambio (Restrepo y Vanegas, 2010), la distancia física y geográfica (Chen y Wang, 2010; Torres y Rendón, 2013), la ubicación geográfica mundial (Venables, 2001), las barreras no arancelarias (Miečinskienè et al., 2014; Baena, 2018), la competitividad en el mercado de destino (Chabowski, 2017) y por último el índice de globalización (Gygli et al., 2018). Por lo tanto, según todo lo anterior, se complementaron entonces los factores generales ya establecidos al agregar nuevos en cada uno. De igual modo, es válido agregar que en el proceso metodológico se consideró para la variable 'precio en destino', tanto los precios de los productos en los mercados exteriores como el nivel de demanda implícito que cada mercado presenta respecto a la adquisición de dichos bienes, tal y como ha sido abordado por otros autores (Roberts et al., 2017). De acuerdo con lo anterior, en la presente versión de la metodología de SMI, la estructura de variables está compuesta por 5 dimensiones generales y 23 subfactores, como se aprecia en la Tabla 1.

Tabla 1: Fuentes de información para la recolección de sub-factores de estudio. (Adaptado de Baena et al., 2018).

\begin{tabular}{|c|c|c|c|}
\hline Factores generales & Sub-factores & Sigla & Fuente de consulta \\
\hline \multirow{5}{*}{ Costos } & Precio en destino & PED & https://bit.ly/31ORDMQ \\
\hline & Costo transporte internacional & CTI & https://bit.ly/2KAOTx7 \\
\hline & Costo de importación & CDI & https://bit.ly/2xd266E \\
\hline & Transporte interno de origen & $\mathrm{TIO}$ & https://bit.ly/2kflyt7 \\
\hline & Tipo de cambio & TDC & https://bit.ly/2KBhfaN \\
\hline \multirow{5}{*}{ Logística } & Tiempo de tránsito & TDT & https://bit.ly/2oCbjjw \\
\hline & Frecuencia de envío & FDE & https://bit.ly/2fDFtgW \\
\hline & Distancia física y geográfica & DFG & https://bit.ly/2LdfG2f \\
\hline & Índice de desempeño logístico & IDL & https://bit.ly/2mDjxrn \\
\hline & Ubicación geográfica mundial & UGM & https://bit.ly/2CQ0CVG \\
\hline \multirow{5}{*}{ Barreras comerciales } & Barreras arancelarias & BAS & https://bit.ly/31QWOf8 \\
\hline & Barreras no arancelarias & BNA & https://bit.ly/2ZDTYs2 \\
\hline & Índice de libertad económica & IDE & https://herit.ag/2K5N3Qe \\
\hline & Competitividad mercado de destino & CMD & https://bit.ly/2yM7uhl \\
\hline & Proteccionismo en general & PEG & https://bit.ly/31RM14r \\
\hline \multirow{4}{*}{ Económico } & Riesgo en país & REP & https://bit.ly/2TPGDK5 \\
\hline & Índice de precios al consumidor & IPC & https://bit.ly/31Rc4ZE \\
\hline & PIB per cápita & PPC & https://bit.ly/2zYbxuB \\
\hline & Tasa de desempleo & TDD & https://bit.ly/2oXfl1M \\
\hline \multirow{4}{*}{ Entorno y cultura } & Facilidad para hacer negocios & $\mathrm{FHN}$ & https://bit.ly/2IHANII \\
\hline & Índice de corrupción & IDC & https://bit.ly/2BJaDBF \\
\hline & Índice de globalización & IDG & https://bit.ly/2PJchM1 \\
\hline & Des-afinidad cultural & DAC & https://bit.ly/2yXmATb \\
\hline
\end{tabular}

\section{La técnica de jerarquía analítica y su aplicación}

El proceso de análisis jerárquico se ha convertido en un método de amplia aplicabilidad en distintos campos del conocimiento y su utilización ha cubierto tópicos que van desde la selección, la evaluación, el análisis costo-beneficio, la asignación, la planeación y ejecución, la jerarquización, la toma de decisiones hasta los pronósticos (Vaidya y Kumar, 2006). En particular en las áreas económico-administrativo ha mostrado ser una herramienta útil en la ponderación de criterios y selección de alternativas para sustentar diverso tipo de decisiones (Ho, 2008; de FSM Russo y Camanho, 2015; Emrouznejad y Marra, 2017). Si bien no es una técnica reciente, su utilidad radica en la contextualización específica en el campo de los negocios, así como en el uso pedagógico para mostrar un proceso de elección concreto basado en criterio experto y fundamentación matemática. Es de resaltar que, en el plano específico de la internacionalización empresarial, la metodología AHP ha sido aplicada en la explicación de los factores de éxito de la industria de servicios a la hora de desarrollar mercados internacionales (Chen y Wang, 2010).

Ahora bien, en el caso específico de la SMI, tras la identificación de las categorías de análisis, esta fase requiere que los estudiantes diseñen el modelo AHP, en donde se busca jerarquizar los juicios emitidos en cada una de las dimensiones previamente establecidas. La importancia del uso de este modelo se deriva de su utilidad para la resolución de problemas cuando existen múltiples criterios y alternativas. En este sentido, esta herramienta determina la importancia que los empresarios le otorgan a las variables en los distintos niveles, donde se usa la resolución numérica de los valores y vectores propios de acuerdo con lo propuesto 
por Saaty (2004) como lo muestra la Tabla 2 y por último los agrupa usando la media geométrica debido a la diversidad de criterios de los empresarios.

Tabla 2: Proceso para la aplicación del modelo AHP. (Adaptado de Saaty, 2004).

\begin{tabular}{|c|c|c|c|}
\hline $\begin{array}{l}\text { Elaboración de una matriz pareada para comparar } \\
\text { cada dimensión a nivel general y local. }\end{array}$ & $A=\left[\mathrm{a}_{\mathrm{ij}}\right]\left[\begin{array}{cccc}1 & \mathrm{a}_{12} & \cdots & \mathrm{a}_{1 \mathrm{n}} \\
\mathrm{a}_{21} & 1 & \cdots & \mathrm{a}_{2 \mathrm{n}} \\
\vdots & \vdots & \ddots & \vdots \\
\mathrm{a}_{\mathrm{n} 1} & \mathrm{a}_{1 \mathrm{n} 2} & \cdots & 1\end{array}\right]$ & & (1) \\
\hline $\begin{array}{l}\text { Solución del sistema matricial a través del uso de los } \\
\text { valores y vectores establecidos, con el objetivo de } \\
\text { obtener los pesos establecidos de las variables en } \\
\text { cada grupo y subgrupo. }\end{array}$ & $\begin{array}{l}W=\left[\begin{array}{cccc}\frac{W 1}{W 1} & \frac{W 1}{W 2} & \cdots & \frac{W 1}{W n} \\
\frac{W 2}{W 1} & \frac{W 2}{W 2} & \cdots & \frac{W 2}{W n} \\
\vdots & \vdots & \ddots & \vdots \\
\frac{W n}{W 1} & \frac{W n}{W 2} & \cdots & \frac{W n}{W n}\end{array}\right] \\
A \times K=\left[\begin{array}{cccc}1 & a_{12} & \cdots & a_{1 n} \\
a_{21} & 1 & \cdots & a_{2 n} \\
\vdots & \vdots & \ddots & \vdots \\
a_{n 1} & \cdots & a_{1 n 2} & 1\end{array}\right] X \\
\lambda \max =\left(\frac{1}{m}\right) \times\left(\frac{W^{\prime} 1}{W_{1}}+\frac{W^{\prime} 2}{W^{\prime} 2}+\cdots+\frac{W^{\prime} n}{W_{n}}\right)\end{array}$ & {$\left[\begin{array}{c}\mathrm{W} \\
\mathrm{W}_{2} \\
\vdots \\
\mathrm{W}_{\mathrm{n}}\end{array}\right]=\left[\begin{array}{c}W^{\prime}{ }_{1} \\
W_{2}^{\prime} \\
\vdots \\
W_{n}^{\prime}\end{array}\right]$} & $\begin{array}{l}\text { (3) } \\
\text { (4) }\end{array}$ \\
\hline $\begin{array}{l}\text { Implementación del índice de consistencia, tasa y } \\
\text { tasa de aleatoriedad, para el análisis de la } \\
\text { congruencia de las evaluaciones otorgadas por los } \\
\text { expertos. }\end{array}$ & $\mathrm{IC}=\frac{\lambda \max -\mathrm{n}}{\mathrm{n}-1}, \mathrm{TC}=\frac{\mathrm{IC}}{\mathrm{IA}}$ & & (5) \\
\hline $\begin{array}{l}\text { Utilización de la media geométrica para agrupar y } \\
\text { suavizar las calificaciones, teniendo en cuenta las } \\
\text { diferentes perspectivas empresariales. }\end{array}$ & $a_{i j}^{\text {Global }}=\prod_{k=1}^{m}\left(a_{i j}^{k}\right)^{a_{k}}$ & & (6) \\
\hline
\end{tabular}

\section{Proceso de selección de la muestra}

En esta fase de la metodología de enseñanza en SMI, es necesario que los estudiantes cuenten con acceso a estadísticas e indicadores de comercio internacional actualizadas y oficiales, con el objetivo de que desde allí se puedan extraer datos cualitativos y cuantitativos confiables, véase Tabla 1 , referentes al proceso exportador de las empresas, y que sea posible determinar un tamaño muestral para su estudio.

En este caso, la información fue extraída de la base de datos de la Dirección de Impuestos y Aduanas Nacionales DIAN (2018) en Colombia, consultando su sitio web en el apartado "Consultas predefinidas de las estadísticas de comercio exterior- SIEX" en cuyo caso fue posible rastrear el total de las exportaciones realizadas en el departamento de Antioquia en el último año de referencia y filtrándolas por el capítulo arancelario que representa los productos químicos (número 38), para así, poder obtener aquellos exclusivamente del sector bajo análisis, dando como resultado que 100 empresas que realizaron transacciones internacionales durante el periodo estudiado. Posteriormente, las cifras fueron ordenadas y agrupadas en tres segmentos (alta, media y baja exportación), con el fin de que la muestra estuviera conformada por opiniones equitativas de cada segmento y poder tomar una muestra aleatoria no intencionada de 10 empresas para el estudio, como se expresa en la Tabla 3.

\section{Recolección de la información}

El proceso de recolección de la información se llevó a cabo a través de entrevistas telefónicas y cuestionarios electrónicos realizados a las personas encargadas del área de comercio internacional o negocios internacionales en las empresas, con el fin de que sus juicios estuviesen fundamentados en la experiencia que han tenido en el ámbito específico y en las prioridades que cada empresa tenía al exportar. Dicho cuestionario, fue diseñado usando la escala numérica propuesta por Saaty (2004) para comparar entre sí las dimensiones generales y los grupos de variables locales, siendo 1 (igual de importante) a 9 (absolutamente más importante) como lo muestra la Tabla 4. 
Tabla 3: Caracterización exportadora de las empresas estudiadas. (Datos tomados de DIAN, 2018).

\begin{tabular}{|c|c|c|c|c|c|}
\hline Empresas & $\begin{array}{c}\text { FOB 2018 } \\
(\text { USD) }\end{array}$ & Países & Empresas & $\begin{array}{c}\text { FOB 2018 } \\
(U S D)\end{array}$ & Países \\
\hline Empresa 1 & $\$ 246.650$ & Estados Unidos & Empresa 6 & $\$ 26.992$ & Turquía \\
\hline Empresa 2 & $\$ 224.595$ & Honduras & Empresa 7 & $\$ 24.283$ & Ecuador \\
\hline Empresa 3 & $\$ 93.109$ & Ecuador & Empresa 8 & $\$ 9.468$ & Uruguay \\
\hline Empresa 4 & $\$ 47.795$ & Ecuador & Empresa 9 & $\$ 9.075$ & Ecuador \\
\hline Empresa 5 & $\$ 27.842$ & Ecuador & Empresa 10 & $\$ 4.617$ & Perú \\
\hline
\end{tabular}

Tabla 4: Escala de valoración de los cuestionarios. (Adaptada de Saaty, 2004).

\begin{tabular}{|c|c|c|c|c|}
\hline \multicolumn{5}{|c|}{ Si la alternativa X es ... Y } \\
\hline 1 & 3 & 5 & 7 & 9 \\
\hline $\begin{array}{l}\text { Igualmente } \\
\text { importante }\end{array}$ & $\begin{array}{c}\text { Apenas más } \\
\text { importante }\end{array}$ & Bastante importante & $\begin{array}{c}\text { Mucho más } \\
\text { importante }\end{array}$ & $\begin{array}{c}\text { Absolutamente } \\
\text { importante }\end{array}$ \\
\hline
\end{tabular}

\section{Determinación de los mercados más viables}

En cuanto a los países destinos, después de obtener la valoración a nivel general y local de los criterios, éstos se evaluaron tomando como referencia los mercados a los que cada empresa efectivamente exportaba, usando como criterios de evaluación el desempeño reflejado en las 5 dimensiones principales, anteriormente mencionadas en la Tabla 1. De esta forma, se buscó que la evaluación de las empresas fuese dirigida a los países a los que exportan actualmente, para fundamentar sus juicios en las experiencias adquiridas durante el proceso de expansión hacia nuevos mercados, con el fin de resaltar el tipo de destinos que representaban mayores ventajas para el sector químico antioqueño. A diferencia de la escala de medición de 1 a 9 propuesta en la Tabla 4, en esta fase la evaluación se encuentra basada en el desempeño que cada mercado presenta en las 5 dimensiones generales, siguiendo la escala Liberatore (1987) de adaptación al modelo AHP, donde se propone como escala de calificación: (A) si las condiciones son adecuadas para exportar, (M) si las condiciones son medianamente adecuadas para exportar y, por último, (I) si las condiciones son inadecuadas para exportar. Dicho cambio en la calificación, se realizó por dos razones: i) para reducir el número necesario de comparaciones a realizar, y ii) para obtener una valoración concreta que determinara las características del mercado, dando una mejor visión del contexto de los mercados antes de incursionar en ellos.

\section{RESULTADOS Y DISCUSIÓN}

La propuesta de enseñanza de mercados internacionales en el presente estudio aplicó una metodología de ayuda fundamentada, que, si bien aplica inicialmente a los estudiantes de Negocios Internacionales, se puede extender a los empresarios, con el fin de fundamentar la toma de decisiones a partir de las prioridades de un sector empresarial determinado, conociendo las ventajas y desventajas que representan sus mercados actuales e identificando los aspectos prioritarios al momento de seleccionar un destino en el exterior. Tomando como ejemplo el caso del sector químico en Antioquia, se estableció la jerarquía de las dimensiones generales y específicas a partir del uso del modelo AHP aplicado a los juicios emitidos por los expertos. De esta manera, la Tabla 5 refleja que los resultados obtenidos establecen el orden de importancia que los empresarios otorgaron a los aspectos en sus distintos niveles, en donde el ranking de las dimensiones generales fue liderado por el aspecto de los costos, seguido de la logística implícita en los procesos, las barreras comerciales, el factor económico del mercado objetivo, y la afinidad con el entorno y la cultura.

Cabe resaltar, que los resultados obtenidos muestran que el factor costos juega un papel determinante para la selección de un mercado internacional en el sector químico, característica que también fue destacada en los resultados del ranking global de las 23 variables, en donde las 5 principales fueron: precio en destino, tiempo de tránsito, costo transporte internacional, costo de importación y barreras arancelarias. De lo anterior se puede inferir, que las decisiones del sector estarían supeditadas a la favorabilidad de los costos que represente un determinado destino. En este caso, al analizar los destinos de exportación actuales de las empresas estudiadas, los datos indican que la mayoría de los destinos fueron países ubicados en América Latina, que presentan estándares y condiciones similares a Colombia, significando menos restricciones para la exportación de productos químicos y siendo mercados atractivos para las empresas del sector como se aprecia en la Figura 1. Finalmente, la calificación de las condiciones para la exportación en estos países reflejó que destinos como Ecuador, Estados Unidos y Honduras presentan unas condiciones de mayor favorabilidad, mientras que otros como Turquía, Perú y Uruguay se destacaron por presentar condiciones regulares y no tan favorables como lo muestra la Tabla 6 . A partir del análisis de los resultados y valoraciones 
anteriores, se logra conocer tanto la importancia de las diferentes dimensiones incidentes en la selección de mercados como el tipo de perfiles que presentan mejores condiciones para la exportación de productos químicos, ampliando la visión de los empresarios y estudiantes al determinar la clase de mercados que requiere el sector para expandir su portafolio de productos adecuadamente.

Tabla 5: Jerarquía de las dimensiones generales y específicas.

\begin{tabular}{|c|c|c|c|c|c|c|c|}
\hline Factores generales & $\begin{array}{c}\text { Pesos } \\
\text { globales }\end{array}$ & Ranking & $\begin{array}{c}\text { Sub- } \\
\text { factores }\end{array}$ & $\begin{array}{l}\text { Pesos } \\
\text { locales }\end{array}$ & $\begin{array}{c}\text { Ranking } \\
\text { local }\end{array}$ & Ponderación & $\begin{array}{c}\text { Ranking } \\
\text { global }\end{array}$ \\
\hline \multirow{5}{*}{ Costos } & \multirow{5}{*}{0,3763} & \multirow{5}{*}{1} & PED & 0,2777 & 1 & 0,1045 & 1 \\
\hline & & & CTI & 0,2084 & 2 & 0,0784 & 3 \\
\hline & & & CDI & 0,1992 & 3 & 0,075 & 4 \\
\hline & & & TIO & 0,1600 & 4 & 0,0602 & 6 \\
\hline & & & TDC & 0,1548 & 5 & 0,0583 & 7 \\
\hline \multirow{5}{*}{ Logística } & \multirow{5}{*}{0,2545} & \multirow{5}{*}{2} & TDT & 0,3995 & 1 & 0,1017 & 2 \\
\hline & & & FDE & 0,2281 & 2 & 0,0581 & 8 \\
\hline & & & DFG & 0,1752 & 3 & 0,0446 & 10 \\
\hline & & & IDL & 0,1058 & 4 & 0,0269 & 15 \\
\hline & & & UGM & 0,0914 & 5 & 0,0233 & 17 \\
\hline \multirow{5}{*}{$\begin{array}{c}\text { Barreras } \\
\text { comerciales }\end{array}$} & \multirow{5}{*}{0,2019} & \multirow{5}{*}{3} & BAS & 0,2993 & 1 & 0,0604 & 5 \\
\hline & & & BNA & 0,2705 & 2 & 0,0546 & 9 \\
\hline & & & IDE & 0,1638 & 3 & 0,0331 & 13 \\
\hline & & & CMD & 0,1359 & 4 & 0,0274 & 14 \\
\hline & & & PEG & 0,1304 & 5 & 0,0263 & 16 \\
\hline \multirow{4}{*}{ Económico } & \multirow{4}{*}{0,1148} & \multirow{4}{*}{4} & REP & 0,3736 & 1 & 0,0429 & 11 \\
\hline & & & IPC & 0,3678 & 2 & 0,0422 & 12 \\
\hline & & & PPC & 0,1321 & 3 & 0,0152 & 20 \\
\hline & & & TDD & 0,1265 & 4 & 0,0145 & 21 \\
\hline \multirow{4}{*}{ Entorno y cultura } & \multirow{4}{*}{0,0526} & \multirow{4}{*}{5} & FHN & 0,3991 & 1 & 0,021 & 18 \\
\hline & & & IDC & 0,2984 & 2 & 0,0157 & 19 \\
\hline & & & IDG & 0,1601 & 3 & 0,0084 & 22 \\
\hline & & & DAC & 0,1424 & 4 & 0,0075 & 23 \\
\hline
\end{tabular}

Tabla 6: Moda correspondiente a las valoraciones recibidas de cada destino.

\begin{tabular}{|l|c|c|c|c|c|c|}
\hline Variables/ Países de destino & Ecuador & $\begin{array}{c}\text { Estados } \\
\text { Unidos }\end{array}$ & Honduras & Turquía & Perú & Uruguay \\
\hline Costos & $\mathrm{A}$ & $\mathrm{A}$ & $\mathrm{A}$ & $\mathrm{M}$ & $\mathrm{M}$ & $\mathrm{A}$ \\
\hline Barreras comerciales & $\mathrm{A}$ & $\mathrm{A}$ & $\mathrm{A}$ & $\mathrm{M}$ & $\mathrm{M}$ & $\mathrm{A}$ \\
\hline Logística & $\mathrm{A}$ & $\mathrm{A}$ & $\mathrm{A}$ & $\mathrm{A}$ & $\mathrm{M}$ & $\mathrm{I}$ \\
\hline Entorno y cultura & $\mathrm{A}$ & $\mathrm{A}$ & $\mathrm{A}$ & $\mathrm{A}$ & $\mathrm{M}$ & $\mathrm{A}$ \\
\hline Económico & $\mathrm{A}$ & $\mathrm{A}$ & $\mathrm{A}$ & $\mathrm{A}$ & $\mathrm{M}$ & $\mathrm{A}$ \\
\hline
\end{tabular}

\section{DISCUSIÓN FINAL}

Considerando la importancia que actualmente tiene la enseñanza de procesos pedagógicos que desarrollen competencias en los estudiantes, y la relevancia que tiene la internacionalización para las empresas, este estudió planteó una propuesta de enseñanza enfocada en la selección de destinos a partir del uso de la aplicación del modelo AHP, actuando como una herramienta práctica para el aprendizaje del alumnado del campo de estudio de negocios internacionales y como una forma de instrumentalizar la toma de decisiones en las organizaciones. Con el fin de demostrar su aplicabilidad, se tomó el caso del sector químico en el departamento de Antioquia (Colombia), para así evidenciar una perspectiva real de los empresarios frente a las oportunidades existentes en los mercados externos y definir cuál dimensión representaba mayor importancia para ellos.

Los resultados obtenidos a partir del modelo, demostraron que la visión de las organizaciones se encuentra fuertemente influenciada por los costos que se incurren para llegar a cada mercado y por la logística implícita 
en los procesos de transporte, pero que aun así, las valoraciones también destacaron que existen varios mercados en Latinoamérica que presentan condiciones favorables bien sea para continuar desarrollando sus operaciones comerciales o para su incursión inicial en éstos, dejando claro que si el sector busca llegar a mercados más exigentes como Europa, deberá desarrollar una estructura financiera que soporte los costos de la internacionalización, así como otras estrategias de apalancamiento.

Desde el punto de vista empresarial, se encuentra los perfiles de países que se perciben más adecuados al momento de exportar desde la experiencia del sector, al igual que se logra establecer un patrón del desempeño de los productos químicos en los diferentes mercados. Luego, no se trata solo de distinguir las prioridades fundamentadas en los juicios empresariales, sino también comprender la perspectiva empresarial dentro del proceso de toma de decisiones alusivas a la selección de posibles compradores, actuando como guía para las empresas del sector que aún no han comenzado su proceso de expansión o para aquellas que quieren expandir su portafolio de clientes externos. Desde la perspectiva académica, los resultados revelan que la aplicación metodológica se puede utilizar en cualquier sector para contrastar los hallazgos, sirviendo como estrategia para impulsar la innovación de los métodos de enseñanza y enfocar el aprendizaje de manera práctica, a través de la obtención de habilidades competitivas, la motivación por la investigación y la resolución de los problemas que atañen a los sectores empresariales.

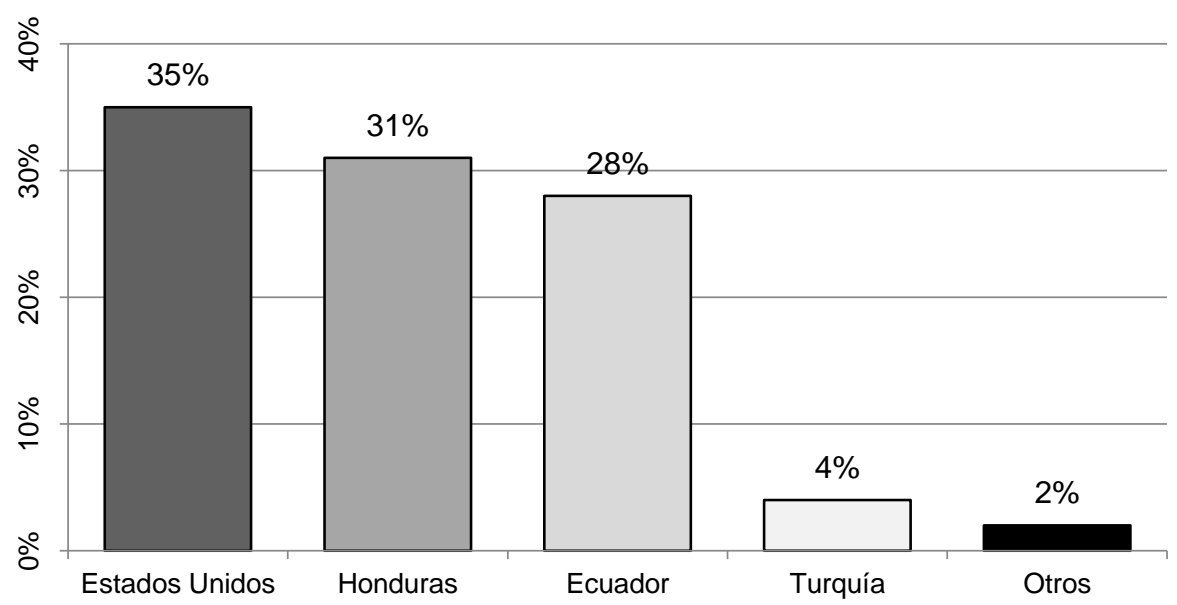

Fig. 1: Principales mercados de las firmas estudiadas. (Datos tomados de DIAN, 2018).

\section{CONCLUSIONES}

De acuerdo a la metodología presentada y a los resultados obtenidos, se pueden extractar cuatro grandes conclusiones principales:

1. La aplicación del modelo AHP en las dimensiones estructuradas en este estudio permite jerarquizar los factores y sub-factores que presentan mayor importancia en la escogencia de mercados foráneos, actuando como una herramienta aplicable durante los procesos de investigación de nuevos destinos.

2. La metodología presenta resultados congruentes con el comportamiento exportador del sector y su aplicación es intuitiva para mejorar los procesos de enseñanza en los negocios, mercadeo internacional y otras áreas afines.

3. La valoración de los mercados a partir de la experiencia de los empresarios, ayuda a identificar un patrón común entre las organizaciones del sector químico antioqueño al momento de considerar las condiciones para la exportación a un destino en específico, siendo entonces un paso a considerar dentro del proceso de la SMI.

4. En líneas futuras de trabajo en la identificación de otros mercados de exportación, teniendo en cuenta los pesos ya establecidos, se podrían incluir otras técnicas analíticas para robustecer el análisis y encontrar otros destinos adicionales para su exploración.

\section{REFERENCIAS}

Akgün, A., H. Keskin, y H. Ayar, Standardization and Adaptation of International Marketing Mix Activities: A Case SMI, doi: 10.1016/j.sbspro.2014.09.080, Procedia-Social and Behavioral Sciences, 150, 609-618 (2014).

Baena, J., Barreras Arancelarias y No Arancelarias como Restricciones al Comercio Internacional, ISSN: 13159984, Revista Venezolana de Gerencia, 23(83), 543-562 (2018). 
Baena, J., J. Cano, y E. Campo, Metodología para la Selección de Mercados Internacionales: Un Análisis de Caso para la Exportación de Bebidas Carbonatadas. Dirección y Organización, ISSN: 1132-175X, (66), 5-16 (2018).

Barber, J., y Darder, F. Dirección de Empresas Internacionales. Pearson Educación. Madrid, España (2004).

Bayo-Moriones, A., M. Billón, y F. Lera-López, Perceived Performance Effects of ICT in Manufacturing SMEs, doi: 10.1108/02635571311289700, Industrial Management \& Data Systems, 113(1), 117-135 (2013).

Brouthers, K., y J. Hennart, Boundaries of the Firm: Insights from International Entry Mode Research, doi: 10.1177/0149206307300817, Journal of Management, 33(3), 395-425 (2007).

Cameron, M., W. Viviers, y Steenkamp, E., Breaking the 'Big Data' Barrier when Selecting Agricultural Export Markets: an Innovative Approach, doi: 10.1080/03031853.2017.1298456, Agrekon, 56(2), 139-157 (2017).

Cano, J., E. Campo, y J. Baena, Application of DEA in International Market Selection for the Export of Goods, doi: 10.15446/dyna.v84n200.63612, DYNA, 84 (200), 376-382 (2017).

Canova, F., y G. De Nicolo, Monetary Disturbances Matter for Business Fluctuations in the G-7, doi: 10.2139/ssrn.231854 Journal of Monetary Economics, 49(6), 1131-1159 (2002).

Chabowski, B. A., Review of Global Competitiveness Research: Past Advances and Future Directions, doi: 10.1509/jim.16.0053 Journal of International Marketing, 25(4), 1-24 (2017).

Chen, M., y S. Wang, The Critical Factors of Success for Information Service Industry in Developing International Market: Using Analytic Hierarchy Process (AHP) Approach, doi:10.1016/j.eswa.2009.06.012, Expert Systems with Applications, 37(1), 694-704 (2010).

Cipoletta, G., Pérez, G., y R. Sánchez, Políticas Integradas de Infraestructura, Transporte y Logística: Experiencias Internacionales y Propuestas Iniciales. Serie Recursos Naturales e Infraestructura No.150. CEPAL (2010).

Crittenden, V., y E. Wilson, Content, Pedagogy, and Learning Outcomes in the International Marketing Course, doi: 10.1300/J066v17n01_05, Journal of Teaching in International Business, 17(1-2), 81-101 (2006).

de FSM Russo, R., y R. Camanho, Criteria in AHP: A Systematic Review of Literature, doi.org/10.1016/j.procs.2015.07.081, Procedia Computer Science, 55, 1123-1132 (2015).

De la Hoz, E., A. González, y A. Santana, Metodología de Medición del Potencial Exportador de las Organizaciones Empresariales, doi: 10.4067/S0718-07642016000600003, Información Tecnológica 27(6), 11-18 (2016).

Dirección de Impuestos y Aduanas Nacionales DIAN. Consultas predefinidas de las estadísticas de comercio exterior SIEX. Obtenido de Estadísticas de Comercio Exterior, Enero-Diciembre (2018).

Emrouznejad, A., y M. Marra, The state of the Art Development of AHP (1979-2017): a Literature Review with a Social Network Analysis, doi.org/10.1080/00207543.2017.1334976, International Journal of Production Research, 55(22), 66536675 (2017)

Fuerst, S., The Development of International Business Theory: Implications for International Business Teaching and Research in Colombia, Cuadernos de Administración (Universidad del Valle), ISSN: 0120-4645, (43), 33-51 (2010).

Gygli, S., Haelg, F., Potrafke, N. y J. Sturm., The KOF globalisation index-revisited, doi: 10.1007/s11558-019-09344-2, The Review of International Organizations, 1 (1), 1-32 (2018).

Ho, W., Integrated Analytic Hierarchy Process and its Applications-A Literature Review, doi.org/10.1016/j.ejor.2007.01.004, European Journal of Operational Research, 186(1), $211-228$ (2008).

Kaufman, P., H. Melton, y otros cuatro autores, Alternative Approaches for Educating Future Global Marketing Professionals: A Comparison of Foreign Study and Research-Intensive Marketing Programs, doi: 10.1177/0273475311420235, Journal of Marketing Education, 33(3), 285-294 (2011).

Kox, H., y H. Rojas-Romagosa, Exports and Productivity Selection Effects for Dutch Firms, doi: 10.1007/s10645-010-91470, De Economist, 158(3), 295-322 (2010).

Lanzas, J. y E. Moral, Mercados Estratégicos para la Promoción del Aceite de Oliva Virgen Español, Revista de Estudios Empresariales, Segunda Época, ISSN: 1988-9046, (1), 85-102 (2010).

Leandro, A., El Proceso de Internacionalización de Empresas, TEC Empresarial, ISSN: 1659-3359, 3(3), 18-25 (2009).

Li, T., C. Chao, y F. Li, Teaching International Marketing in an Experiential Learning Course: Practice and Impacts, International Journal of Education Research, ISSN: 1932-8443, 8(1), 37-48. 12p (2013).

Liberatore, M., An Extension of the Analytic Hierarchy Process for Industrial R\&D Project Selection and Resource Allocation, doi: 10.1109/TEM.1987.6498854, Engineering Management, IEEE Transactions, 34(1), 12-18 (1987).

London, K., Multi-market Industrial Organizational Economic Models for the Internationalization Process by Small and Medium Enterprise Construction Design Service Firms, doi: 10.3763/aedm.2009.0111, Architectural Engineering and Design Management, 6(2), 132-152 (2010).

Martelo, R., I. Jiménez-Pitre y A. Quintana, Determinación del Perfil Profesional de Estudiantes de Pregrado Aplicando la Técnica de Análisis Comparativo, doi: 10.4067/S0718-07642018000200029 Información Tecnológica, 29(2), 29-40 (2018). 
Martín, O., y R. Drogendijk, Country Distance (COD): Development and Validation of a New Objective Measure. Journal of Small Business Management, doi: 10.1111/jsbm.12035, 52(1), 102-125 (2014).

Miečinskienè, A., V. Stasytytè, y J. Kazlauskaitè, Reasoning of Export Market Selection, doi: 10.1016/j.sbspro.2013.12.963, Procedia-Social and Behavioral Sciences, 110, 1166-1175 (2014).

Muduli, A., V. Kaura, y A. Quazi, Pedagogy or Andragogy? Views of Indian Postgraduate Business Students, doi: 10.1016/j.iimb.2018.01.008, IIMB Management Review, 30(2), 168-178 (2018).

Nakos, G. y L. Brouthers, The Role of Systematic International Market Selection on Small Firms' Export Performance, doi: 10.1111/j.1540-627X.2005.00142.x, Journal of Small Business Management, 43(4), 363-381 (2005).

Papadopoulos, N., y J. Denis, Inventory, Taxonomy and Assessment of Methods for International Market Selection, doi: 10.1108/eb008357, International Marketing Review, 5(3), 38-51 (1988).

Rahman, S., Organisational and Strategic Considerations in International Market Selection, International Journal of Applied International Business, ISSN: 2614-7432, 1(2), 1-14 (2006).

Restrepo, J. y J. Vanegas, Competitividad y Comercio Exterior de las Pymes del Sector Textil-confecciones del Valle de Aburrá: Incidencia del Tipo de Cambio, ISSN: 0124-5821, Revista Virtual Universidad Católica del Norte, 1(30), 185-204 (2010).

Roberts, M., D. Xu, X. Fan, y S. Zhang, The Role of Firm Factors in Demand, Cost, and Export Market Selection for Chinese Footwear Producers, doi: 10.3386/w17725, The Review of Economic Studies, 85(4), 2429-2461 (2017).

Ruíz, A., Educación Superior y Globalización Educar, ¿para qué? pp. 237 Ciudad de México. Editorial Plaza y Valdéz (2013).

Saaty, T., Decision making - the Analytic Hierarchy and Network Processes (AHP/ANP), doi: 10.1007/s11518-006-01515, Journal of Systems Science and Systems Engineering, 13(1), 1-35 (2004).

Senna, E., L. dos Santos, L., y R. da Silva, The Challenge of Teaching Business Logistics to International Students, doi: 10.3182/20130911-3-BR-3021.00105, IFAC Proceedings Volumes, 46(24), 463-470 (2013).

Targhi, M., F. Rad, y M. Rezaeezadeh, The Relationship between Firm's Competitiveness and Commitment to Export on the SMEs' Marketing Strategies. Journal of Economic and Management Perspectives, ISSN: 2523-5338, 11(3), 668-680 (2017).

Torres, A. y O. Rendón, El Transporte Internacional como Factor de Competitividad en el Comercio Exterior, doi: 10.1016/S2077-1886(13)70035-0, Journal of Economics Finance and Administrative Science, 18(35), 108-118 (2013).

Vaidya, O. y S. Kumar, Analytic Hierarchy Process: An Overview of Applications, doi.org/10.1016/j.ejor.2004.04.028, European Journal of operational research, 169(1), 1-29 (2006).

Vanegas, J., J. Restrepo, S. Tabares, y E. Anzo, Exportaciones y Pymes en Colombia: un Análisis desde las Características Gerenciales. Capítulo 9. pp. 232-267. En Rincón, Y., Restrepo, J. y Vanegas, J. (Coords.) (2018) Gestión Organizacional y Desarrollo Responsable en las PYME. Una Mirada Glocal. Serie PYME. Tomo IV. Primera Edición. pp. 486. Medellín, Colombia. Sello Editorial PUBLICAR-T. Tecnológico de Antioquia, Institución Universitaria (2018).

Vanegas, J., S. Tabares, E. Anzo, y J. Restrepo, Dinámica Exportadora e Instituciones: Contexto de los Destinos de las Exportaciones Industriales de las Pymes Colombianas pp. 99-124. En Romo Morales, G. (Coord.) (2018). La Empresa como Institución. pp. 178. Guadalajara, México. Universidad de Guadalajara (2018).

Venables, A., Geography and International Inequalities: the Impact of New Technologies. Journal of Industry, doi: 10.1023/A:101283052, Competition and Trade, 1(2), 135-159 (2001).

Vielba, C. y D. Edelshain, Teaching International Business Management Effectively, doi: 10.1108/02621719510100825, Journal of Management Development, 14(10), 32-47 (1995).

Villalobos-Abarca, M., V. Karmelic-Pavlov, y M. Néspolo-Cova, Enseñanza de los Procesos en Ingeniería Software -vsCompetitividad de Empresas Creadas por Ingenieros Informáticos, doi: 10.4067/S0718-50062016000100002, Formación Universitaria, 9(1), 3-14 (2016). 